references are included at the end of each chapter.

The style is still didactic and topic orientated, with most chapters being based on anatomical areas, starting with a brief description of normal structures, followed by subheadings, each with related compact paragraphs; entries are not wordy although since few statistics are cited, some of the statements are imprecise. Occasional oversimplifications are apparent but this is a book aimed at the novice in ocular pathology and other broader based and deeper texts are available to those who are moved to ask for more. The succinct format is complimented by some of the new chapters. I particularily enjoyed the chapter on the lens, a structure oftne all but ignored by light-microscopists and also the chapter on congenital anomalies, which is a useful guide to a difficult and complex subject.

I am often asked by those preparing for the FRCS and College of Ophthalmology examinations, to recommend 'a good textbook, . . . this is it; although it will no longer slip neatly into the pocket of a white coat, it also is not likely to remain unread on the shelf, long after the exams have been passed.

\section{Aids to Ophthalmology}

P. T. Khaw, D. S. Hughes, S. J. Keightley, R. F. Walters, A. R. Elkington

(Churchill Livingstone) 294 pages

Take four enthusiasts, toss in a well-seasoned examiner and the result should be a feast for examination candidates who would indeed be foolish not to buy this concentrated distillate of knowledge.

Anatomical and physiological as well as clinical data are presented in profusion as well as informative sections on relevant medical conditions such as diabetes and the collagen disorders. New material is present; the new WHO classification of trachoma is included. There is a plethora of old exam chestnuts such as the content of a vial of mydricaine, the lethal dose of atropine and visual standards for driving. These and the many other examples are sometimes difficult if not impossible to find in conventional texts.

Fact, and not explanation, is what is contained within these covers. It is an aide- memoire, a crammer, not a textbook. Hence I could not recommend it to medical students and others on the lower slope of the learning curve.

There are a few editorial lapses. Alkali burns and vernal disease are fully covered both in the conjunctival and corneal chapters. Openings such as 'the orbit contains globe, fat, muscles, nerves, vessels and lacrimal gland' seem superfluous. There has been little attempt to make the lists easy to remember or to use tables and diagrams to break the monotony. There are acts of omission e.g. the characteristic angiogram findings in AMPPE and occasionally the negative needs to be stressed; POHS, sandwiched as it is between candiasis and toxoplasmosis, is a condition in which, usually, the vitreous is quiet.

Occasionally the book is somewhat dated: 'trabeculectomy is indicated where there is continuing visual field loss on maximal medical therapy' (? including acetazolamide). Congenital glaucoma is still divided into primary and secondary rather than the trabecular/iridotrabecular/iridocorneal dysgenesis classification from which the clinical findings and embryology can be surmised. The vertical gaze centre may not be as well understood as its horizontal partner but more is known about it than the authors let on and surely the fact that fibres subserving up-gaze cross in the posterior commissure is worth recording. MRI is not mentioned at all, not even a small exam tip on how to differentiate a $\mathrm{T} 1$ from a $\mathrm{T} 2$ weighted image.

Despite these faults this book has much to commend itself to teachers trying to refresh their memory, to clinicians in need of a list of differential diagnoses for a rare disease, as well as to those trying to avoid an embarrassing silence during a viva. In short this is 'teaching time for everyone' and the authors have every right to be 'over the moon' with the result of their considerable endeavours.

Michael Hayward

\section{Review-Dictionary of Eye Terminology}

B. Cassin, S. Solomon

Edited by M. L. Rubin

This soft pocket book contains a small but substantial dictionary of commonly-and not 
so commonly-used ophthalmic terms and abbreviations, and is primarily directed to the North American market. The aim of the authors is to provide a simplified, understandable definition of a large number of ophthalmological terms and in this they have largely succeeded, missing very little out. However this has led to the inclusion of definitions of terms that are scarcely necessary such as eye and postprandial, and eponymous syndromes where the more commonly used name is subtitled. The book also features mis-spellings of terms which, while often amusing, scarcely justify their space in the text. The reader should also be warned that the phonetic pronunciation is American viz 'oculist-ahk-yoolist'. The book is aimed at the ophthalmic technologist, fortunately defined in the text, and would be most useful in this country for medical secretaries. It might usefully be read by medical students, nurses and professionals allied to ophthalmology. There is little new here for the practicing ophthalmologist, but it might prove useful in deciphering some of the more obscure phrases and abbreviations in the American literature.

Miles Stanford 